\title{
Characteristics, Vaccination Status and Outcomes Among Healthy Younger Adults in a Large Public Healthcare System in the South Florida Region
}

\author{
Shenae Samuels ${ }^{1} \cdot$ Jianli Niu $^{1} \cdot$ Candice Sareli $^{1} \cdot$ Aharon Sareli $^{2} \cdot$ Paula Eckardt $^{3}$ (D) \\ Accepted: 7 January 2022 / Published online: 21 January 2022 \\ (c) The Author(s), under exclusive licence to Springer Science+Business Media, LLC, part of Springer Nature 2022
}

\begin{abstract}
Nationally, the 18-49 years old age group are less likely to be vaccinated compared to those 50 years and older. Data describing the risk of COVID-19 severe illness that requires hospitalization among younger healthy adults is limited. In an effort to underscore the importance of vaccination and provide data that may influence COVID-19 risk perception, COVID-19 data of a sample of hospitalized non-elderly age group who clinically may not be considered as high risk for severe COVID-19 illness are presented. Specifically, this retrospective chart review (spanning the period of March 2020 to September 2021) provides a descriptive analysis examining the characteristics, vaccination status and outcomes of adults who were hospitalized at Memorial Healthcare System with laboratory-confirmed COVID-19. The study's data focuses on non-pregnant adults, aged 18-49 years old, without underlying conditions and with no reported history of smoking. As a sub-analysis, data on young and otherwise healthy pregnant females who were hospitalized with COVID-19, as well as data stratified by the pre-Delta and Delta variant dominant period are also presented. There was a total of 482 young and otherwise healthy non-pregnant adults who were hospitalized with COVID-19. Overall, more than $13 \%$ of our study population had severe COVID-19 disease. Further, a higher proportion of unvaccinated patients had severe COVID-19 compared to those who received at least one dose of the vaccine. All ventilator or ECMO placements, 30-day readmissions and deaths occurred among unvaccinated patients.
\end{abstract}

Keywords Infectious disease $\cdot$ SARS-CoV-2 $\cdot$ Vaccination $\cdot$ Hospitalization $\cdot$ Young adults

\section{Introduction}

The novel coronavirus disease 2019 (COVID-19), caused by the Severe Acute Respiratory Syndrome Coronavirus-2 (SARS-CoV-2), is one of the worst infectious disease outbreaks of recent times and continues to be a public health concern within communities across the globe. In the United States (US), the first confirmed case of SARS-CoV-2 was reported on January 31, 2020 in Washington State [1] and

Paula Eckardt

PEckardt@mhs.net

1 Office of Human Research, Memorial Healthcare System, 4411 Sheridan Street, Hollywood, FL 33021, USA

2 Division of Critical Care Medicine, 3501 Johnson Street, Hollywood, FL 33021, USA

3 Division of Infectious Disease, Memorial Healthcare System, 5647 Hollywood Boulevard, Hollywood, FL 33021, USA by March 11, 2020, WHO declared COVID-19 a worldwide pandemic [2]. During the latest wave of COVID-19, fueled by the highly contagious Delta variant, communities saw a drastic rise in COVID-19 cases with younger adults being more represented in cases of severe COVID-19 compared to previous waves. Like communities across the nation, the large and diverse community, served by Memorial Healthcare System (MHS), continues to be afflicted by COVID19. Nationally, Miami-Dade county ranks in the top 3 for the total number of confirmed cases by county with over 676,000 confirmed cases while Broward is in the top 25 with over 358,000 confirmed cases (data as of November 1, 2021) [3]. As it relates to COVID-19 deaths, Miami-Dade ranks in the top 10 counties, nationally, with over 6,470 deaths (data as of November 1, 2021) [3].

To mitigate the risk of COVID-19 infection and the risk of severe disease, vaccination has been recommended by public health experts. As of the writing of this article, almost $80 \%$ of the US adult population had received at least one 
dose of the COVID-19 vaccine, whereas almost $70 \%$ were considered fully vaccinated (data as of November 1, 2021) [4]. While the US has seen increases in the rate of vaccination, vaccine hesitancy continues to be an issue at the forefront in the fight against the COVID-19 pandemic. A larger proportion of younger adults remain vaccine hesitant. Nationally, the non-elderly age group is more likely to be vaccine hesitant and ultimately, unvaccinated. Of the non-elderly age group, those 30-49 years old were the most vaccine hesitant, according to a recent Kaiser Family Foundation (KFF) report [5]. Similarly, data (as of October 29, 2021) from the Florida COVID-19 weekly situation report show that Floridians aged 20-49 years old were less likely to have received at least one dose of the vaccine compared to those 50 years and older $(20-29$ years old: $55 \%$; 30-39 years old: $64 \%$; $40-49$ years old: $73 \%$ vs. $50-59$ years old: $78 \%$; 60-64 years old: 85\%; 65 years and older: 89\%) [6].

Vaccine hesitancy may stem from several factors, including distrust in the medical system, concerns for out-ofpocket costs, as well as immediate or long-term concerns regarding safety and health impacts [5]. Risk perception may also play a key role in vaccine hesitancy and uptake as risk perception may guide decision making in taking preventive action against COVID-19 through vaccination [7]. It has been well-documented that older age and the presence of certain underlying conditions are associated with an increased likelihood of severe COVID-19 illness [8-11]. Further, the risk for older populations and individuals with certain medical conditions has been broadly publicized in the scientific literature, as well as by way of mainstream media. However, data which underscores the risk of severe COVID-19 illness among younger healthy adults (i.e. those without underlying health conditions) is limited and not as widely publicized which may contribute to reduced risk perception among younger healthy adults compared to older adults and/or individuals with underlying conditions that are known to increase the risk of severe COVID-19.

Owusu et al. conducted a study using data from the US Centers for Disease Control and Prevention's (CDC) COVID-19-Associated Hospitalization Surveillance Network (COVID-NET) to describe the epidemiology, characteristics, and outcomes of adults aged 18-49 years without underlying conditions who were hospitalized with COVID19 . Results of their study showed that, during the period of March 2020-August 2020, 22\% of patients were admitted to an intensive care unit, $10 \%$ required mechanical ventilation, and $0.6 \%$ of patients died [12]. The study's data spanned 13 states not inclusive of data from Florida.

Given the high number of cases throughout the state during the summer of 2021, Florida residents were at an exponential risk of COVID-19 exposure. The current study adds to previous findings by providing data pertaining to the characteristics, vaccination status and outcomes of hospitalized
COVID-19 adults, aged 18-49 years old, without underlying conditions and without a history of smoking. Cases described were admitted at a large public healthcare system in the diverse South Florida region; a region not previously represented in the study by Owusu et al. [12]. The overall goal of this study is to provide data that may influence the perceived risk of severe COVID-19 illness among a nonelderly age group, who clinically may not be considered to be high risk for severe COVID-19 illness, in an effort to further underscore the importance of adhering to public health guidelines, including vaccination recommendations.

\section{Methods}

\section{Study Participants and Setting}

Electronic medical records (EMR) of patients who were hospitalized with confirmed SARS-CoV-2 infection at an MHS facility during the period of March 2020 and September 2021 were extracted. The main study population included non-pregnant patients, aged 18 to 49 years old, without underlying medical conditions (see Table S1) and with no history of smoking on record $(n=482)$. As a secondary analysis, the characteristics and outcomes of a sub-population of otherwise healthy (i.e. without a record of underlying conditions and history of smoking) pregnant patients will also be examined $(n=167)$. MHS, which serves the South Florida community, is the third largest public healthcare system in the nation.

\section{Data Sources}

Data points of patients with confirmed SARS-CoV-2 was extracted from the EMR. Data points of this study included patient demographics, underlying disease/co-morbidities on record at time of hospitalization, smoking status, pregnancyspecific encounter, presenting vitals, home medications, signs and symptoms at admission, COVID-19 associated treatment, vaccination status and patient outcomes. The vaccinated group included patients who received one dose of the Janssen vaccine, at least one dose of a messenger RNA (mRNA) vaccine (Pfizer-BioNTech or Moderna), or two full doses of an mRNA vaccine. All others were coded as unvaccinated. In accordance with the privacy requirements of the Health Insurance Portability and Accountability Act (HIPAA), all ages over 89 years old were coded as 90 years old. To categorize the study population based on the severity of their COVID-19 illness, severe cases of COVID-19 were defined as patients who received oxygen supplementation by way of ventilator, extracorporeal membrane oxygenation (ECMO), continuous positive airway pressure/bi-level positive airway pressure (CPAP/BiPAP) or high-flow oxygen. 
Outcome measures included the rates of severe COVID-19, 30-day readmission and death.

\section{Statistical Analysis}

To describe the characteristics and outcomes of the study population, descriptive statistics were calculated for all demographic, clinical variables, and outcomes. Categorical variables are presented as absolute number and frequencies (\%) while continuous variables are presented as medians and interquartile range (IQR) or means and standard deviations (SD). All analyses were performed using Stata/SE 15.1 statistical software.

\section{Results}

Overall, from March 2020 to September 2021, there were a total of 11,882 confirmed COVID-19 hospitalizations at MHS. Adults, aged 18 to 49 years, accounted for $33 \%$ of all COVID-19 hospitalizations during the study period while non-pregnant adults, aged 18 to 49 years without underlying conditions and a history of smoking, accounted for $4 \%$ of all COVID-19 hospitalizations (Table S2). Table 1 presents patient demographics, clinical characteristics and outcomes of the main study population of non-pregnant adults aged 18 to 49 without underlying conditions and no history of smoking. Almost $40 \%$ of COVID-19 hospitalizations that occurred among this main study population were during the Delta variant dominant period of July 2021 to September 2021. Among this young and otherwise healthy population, the median age was 38 years old, $59.8 \%$ were men, $32.4 \%$ were Hispanic, and $69.9 \%$ were privately insured. Over $13 \%$ $(n=65)$ had severe COVID-19, 1.9\% $(n=9)$ were admitted to the intensive care unit (ICU) and 5 patients (1.0\%) died during hospitalization.

The vast majority of the young and otherwise healthy adults who were hospitalized with confirmed COVID-19 were unvaccinated (93.4\%). In describing COVID-19 complications and outcomes by COVID-19 vaccination status (Table 2), a higher proportion of unvaccinated patients had severe COVID-19 compared to those who received at least one dose of the vaccine ( $14.0 \%$ vs. $6.3 \%)$. Further, all patients who were placed on a ventilator or ECMO were unvaccinated $(n=7)$ and all 30 -day readmissions $(n=17)$ occurred among unvaccinated patients. All deaths $(n=5)$ within our study population of young and otherwise healthy non-pregnant adults occurred among unvaccinated patients with a median age of 29 years old.

We also described the vaccination status, COVID-19 complications and outcomes of a sub-population of young and otherwise healthy adults with confirmed COVID-19 who were admitted with a pregnancy-related encounter
Table 1 Patient demographics, clinical characteristics and outcomes of non-pregnant adults aged 18-49 years without underlying medical conditions who were hospitalized with laboratory-confirmed COVID19, March 2020 to September 2021

\begin{tabular}{|c|c|}
\hline Characteristics & $\mathrm{N}(\%)$ or median (IQR) \\
\hline $\mathrm{N}$ & 482 \\
\hline \multicolumn{2}{|l|}{ Delta dominant period } \\
\hline Pre-delta wave (March 2020-June 2021) & $292(60.6)$ \\
\hline Delta wave (July 2021-September 2021) & $190(39.4)$ \\
\hline \multicolumn{2}{|l|}{ Patient characteristics } \\
\hline \multicolumn{2}{|l|}{ Age categories (years) } \\
\hline $18-29$ & $106(22.0)$ \\
\hline $30-39$ & $154(32.0)$ \\
\hline $40-49$ & $222(46.1)$ \\
\hline Age in years, median (IQR) & $38(18-49)$ \\
\hline \multicolumn{2}{|l|}{ Sex } \\
\hline Female & $194(40.3)$ \\
\hline Male & $288(59.8)$ \\
\hline \multicolumn{2}{|l|}{ Race } \\
\hline White & $76(15.8)$ \\
\hline Black & 143 (29.7) \\
\hline Hispanic & $156(32.4)$ \\
\hline Asian & $10(2.1)$ \\
\hline American Indian/Native Hawaiian & $1(0.2)$ \\
\hline Other/multi-racial & $92(19.1)$ \\
\hline Unknown & $4(0.8)$ \\
\hline \multicolumn{2}{|l|}{ Insurance status } \\
\hline Uninsured & $66(13.7)$ \\
\hline Private/managed care & 337 (69.9) \\
\hline Medicaid & $26(5.4)$ \\
\hline Medicare & $24(5.0)$ \\
\hline Other & $29(6.0)$ \\
\hline \multicolumn{2}{|l|}{ Vaccination status } \\
\hline Unvaccinated & $450(93.4)$ \\
\hline Incomplete dose & $6(1.2)$ \\
\hline Fully vaccinated & $26(5.4)$ \\
\hline \multicolumn{2}{|l|}{ Vaccination type } \\
\hline Janssen & $1(3.1)$ \\
\hline Moderna & $5(15.6)$ \\
\hline Pfizer-BioNTech & $26(81.3)$ \\
\hline \multicolumn{2}{|l|}{ Presenting signs and symptoms } \\
\hline Cough & $466(96.7)$ \\
\hline Diarrhea & $46(9.5)$ \\
\hline Systolic blood pressure, median (IQR) & $121(83-163)$ \\
\hline Diastolic blood pressure, median (IQR) & $72(51-105)$ \\
\hline Febrile (temperature $\geq 38^{\circ}$ Celsius) & $241(50.0)$ \\
\hline Heart rate $\geq 100$ beats $/ \mathrm{min}$ & $404(83.8)$ \\
\hline Respiratory rate $>20$ breaths/min & 409 (84.9) \\
\hline \multicolumn{2}{|l|}{ Hospital Course and COVID-19 treatment } \\
\hline $\begin{array}{l}\text { Time (in days) between the onset of symp- } \\
\text { toms and admission, median (IQR) }\end{array}$ & $1(0-27)$ \\
\hline Azithromycin & $51(10.6)$ \\
\hline
\end{tabular}


Table 1 (continued)

\begin{tabular}{ll}
\hline Characteristics & N (\%) or median (IQR) \\
\hline Remdesivir & $74(15.4)$ \\
Systemic corticosteroids & $22(4.6)$ \\
Dexamethasone & $88(18.3)$ \\
Convalescent plasma & $90(18.7)$ \\
Highest level of severity of respiratory illness during hospitalization \\
Death & $5(1.0)$ \\
Ventilator or ECMO & $7(1.5)$ \\
CPAP/BiPAP/high-flow oxygen & $58(12.0)$ \\
Low-flow supplemental oxygen & $303(62.9)$ \\
No supplemental oxygen & $109(22.6)$ \\
ICU Admission & $9(1.9)$ \\
Length of ICU stay (days), median (IQR) & $1(0-5)$ \\
Length of hospital stay (days), median & $4(0-25)$ \\
(IQR) & \\
COVID-19 complications & \\
ARDS & $479(99.4)$ \\
Outcomes & $65(13.5)$ \\
Severe COVID-19 & $17(3.5)$ \\
30-day readmission & $5(1.0)$ \\
Death &
\end{tabular}

Proportions represent column percentages. Proportions may not add to $100 \%$ due to rounding error

$I Q R$ interquartile range, $I C U$ intensive care unit, ECMO extracorporeal membrane oxygenation, $C P A P$ continuous positive airway pressure, BiPAP Bi-level positive airway pressure, $A R D S$ acute respiratory distress syndrome

(Table 3). Of the 11,882 confirmed COVID-19 hospitalizations, $1.4 \%(\mathrm{n}=167)$ was among patients with a pregnancy-related encounter (Table S2). Over 97\% of the sub-population that was hospitalized with COVID-19 was unvaccinated. While no deaths occurred among the sub-population, approximately $2.4 \%(\mathrm{n}=4)$ had severe COVID-19, and $2 \%(n=3)$ were readmitted within 30 days of discharge.

With reports of the more contagious Delta variant affecting younger populations compared to previous strains of COVID-19, we aimed to describe the counts and proportions of younger age groups, as well as the non-pregnant and pregnant otherwise healthy study populations who were hospitalized during the Delta dominant period (beginning in July 2021) compared to the pre-Delta dominant period (Table S2). We also described the rate of severe COVID-19 illness among the main study population of non-pregnant young and healthy adults. Our data show that a higher proportion of the younger age group of 18 to 49 years $(37.3 \%$ vs. $31.5 \%$ ), as well as the study population of young adults without underlying conditions and a history of smoking $(5.7 \%$ vs. $3.4 \%)$ were admitted during the Delta dominant period. The Delta dominant period also had a much higher
Table 2 Severity of respiratory illness, COVID-19 complications and outcomes by COVID-19 vaccination status

\begin{tabular}{lcc}
\hline Characteristics & Unvaccinated (\%) & Vaccinated (\%) \\
\hline $\mathrm{N}$ & $450(93.4)$ & $32(6.6)$ \\
COVID-19 treatment/complications & \\
Highest level of severity of respiratory illness during hospitaliza- \\
$\quad$ tion \\
Death & $5(1.1)$ & $0(0.0)$ \\
$\quad$ Ventilator or ECMO & $7(1.6)$ & $0(0.0)$ \\
CPAP/BiPAP/high-flow & $56(12.4)$ & $2(6.3)$ \\
$\quad$ oxygen & $282(62.7)$ & $21(65.6)$ \\
Low-flow supplemental & & \\
$\quad$ oxygen & $100(22.2)$ & $9(28.1)$ \\
No supplemental oxygen & $448(99.6)$ & $31(96.9)$ \\
ARDS & & $2(6.3)$ \\
Outcomes & $63(14.0)$ & $0(0.0)$ \\
Severe COVID-19 & $17(3.8)$ & $0(0.0)$ \\
30-day readmission & $5(1.1)$ & \\
Death &
\end{tabular}

The vaccinated group includes fully vaccinated patients, as well as those with an incomplete dose (i.e. patients who had at least one dose of a two dose vaccine). Proportions represent column percentages Proportions may not add to $100 \%$ due to rounding error IQR Interquartile range; ICU Intensive Care Unit; ECMO Extracorporeal Membrane Oxygenation; CPAP Continuous Positive Airway Pressure; BiPAP Bi-level Positive Airway Pressure; ARDS Acute Respiratory Distress Syndrome

Table 3 Vaccination status, COVID-19 complications and outcomes among healthy young Adults admitted with a pregnancy-related encounter, March 2020 to September 2021

\begin{tabular}{ll}
\hline Characteristics & $\begin{array}{l}\mathrm{N}(\%) \text { or } \\
\text { median } \\
(\mathrm{IQR})\end{array}$ \\
\hline $\mathrm{N}$ & 167 \\
Vaccination status & $163(97.6)$ \\
Unvaccinated & $1(0.6)$ \\
Incomplete dose & $3(1.8)$ \\
Fully vaccinated & \\
COVID-19 complications & $157(94.0)$ \\
ARDS & \\
Outcomes & $4(2.4)$ \\
Severe COVID-19 & $3(1.8)$ \\
30-day readmission & $0(0.0)$ \\
Death &
\end{tabular}

Proportions represent column percentages. Proportions may not add to $100 \%$ due to rounding error

IQR Interquartile range; ICU Intensive Care Unit; ECMO Extracorporeal Membrane Oxygenation; CPAP Continuous Positive Airway Pressure; BiPAP Bi-level Positive Airway Pressure; ARDS Acute Respiratory Distress Syndrome 
proportion of severe COVID-19 cases among non-pregnant young and healthy adults (17.9\% vs. 10.6\%).

\section{Discussion}

Among a cohort of COVID-19 hospitalizations within a large healthcare system in the South Florida region, 33\% of COVID-19 hospitalizations were among young adults aged 18 to 49 years. Comparatively, individuals aged 18 to 49 years old have made up only $10.5 \%$ to $18.5 \%$ of hospitalizations over the past 4 influenza seasons in the United States, spanning the years 2015-2019 [13] suggesting that otherwise healthy young adults are being more severely impacted by COVID-19 compared to influenza.

In our study, patients aged 18 to 49 years old without underlying conditions and with no history of smoking made up approximately $4 \%$ of COVID- 19 hospitalizations. While death occurred in only $1 \%$ of the study population, over $13 \%$ of the study population had severe COVID-19 based on the measure of having received high supplemental oxygen. Further, a vast majority (99.4\%) of our sample of young and otherwise healthy patients had a diagnosis of acute respiratory distress syndrome (ARDS) noted in their EMR and almost $2 \%$ of patients were admitted to the ICU. While it has been well-documented that the older age groups and individuals with certain underlying conditions are disproportionately at risk for severe COVID-19 [8-10, 14, 15], results of our study further supports the notion that younger and otherwise healthy adults are not immune to COVID-19 severe disease and death. This is especially true during the more contagious Delta dominant period where results of our study show that the younger age group and even those without underlying conditions made up a higher proportion of COVID-19 hospitalizations during the Delta dominant period compared to previous periods with less contagious variants.

Factors which may contribute to severe COVID-19 in younger adults without underlying conditions is limited. Studies have suggested that angiotensin-converting enzyme 2 (ACE2) may play a role in the risk of COVID-19 whereas higher ACE2 levels may facilitate SARS-CoV-2 infection [16]. Studies have further suggested that sex hormones may influence ACE2 levels with males having increased ACE2 levels [17]. As such, the differences in ACE2 levels influenced by sex hormones may explain the increased risk of severe COVID-19 in males as compared to females [16, 17]. In our study, males accounted for almost $60 \%$ of the young and otherwise healthy adults who were hospitalized with COVID-19; providing further support that, despite the absence of underlying conditions, males may be at an increased risk for COVID-19 severe disease.

There is substantial evidence of the disproportionate effect of COVID-19 on people of color [18-21]. Despite making up only $28.6 \%$ and $17.4 \%$ of the population in the counties served by MHS (Broward and Miami-Dade counties, respectively) [22], Blacks accounted for almost $30 \%$ of the study population of young and otherwise healthy individuals who were hospitalized with COVID-19. These results suggest that, even in the absence of underlying conditions, people of color (specifically, Blacks within the communities served by MHS) are disproportionately affected by COVID19 illness requiring hospitalization.

Vaccination continues to be a key recommendation in preventing COVID-19, as well as in reducing the risk of severe COVID-19 and death [23]. A study presenting average weekly incidence (per 100,000 population) data from 13 U.S. jurisdictions found that individuals who were not fully vaccinated were 10 times more likely to have been hospitalized and 11 times more likely to have died from COVID19 compared to fully-vaccinated individuals [24]. Further, despite the prevalence of the more contagious Delta variant, vaccine effectiveness against COVID-19 hospitalizations remain high at $86 \%$; specifically for the currently authorized mRNA vaccines (Pfizer-BioNTech or Moderna) [25]. Our data further supports vaccine effectiveness in reducing hospitalization and death as individuals who received at least one dose of a COVID-19 vaccine accounted for only about $7 \%$ of our sample of COVID-19 hospitalizations whereas unvaccinated patients accounted for approximately 93\% among our sample of hospitalized young and otherwise healthy adults. None of the vaccinated patients in our cohort required ventilator or ECMO placements. Further, all deaths within our study population occurred among patients who were unvaccinated. Despite the plethora of evidence regarding vaccine effectiveness, vaccine hesitancy continues to be a significant barrier to COVID-19 vaccine uptake. A report by Kaiser Family Foundation (KFF) shows that the 18-49 years old age group are least likely to be vaccinated compared to those 50 years and older ( $44 \%$ vs. $56 \%$ ) with over $60 \%$ of those $18-49$ years old reporting that they will "definitely not get the vaccine" compared to $34 \%$ for those 50 years and older. Further, a far smaller proportion of Blacks (11\%) and Hispanics (15\%) are vaccinated compared to Whites (64\%) [5]. In addition, compared to vaccinated individuals, the report also shows that unvaccinated individuals are far more likely to believe that the gravity of COVID-19 is overstated (22\% vs. $57 \%$ ) and they are also less likely to worry about getting sick from COVID-19 (33\% vs. $26 \%$ ) [5]. Undoubtedly, vaccine hesitancy and the lack of vaccine uptake among younger adults and people of color have influenced the observed results of our study. Coupled with the lack of vaccine uptake, previous data suggestive of a reduced perceived risk of COVID-19 among the unvaccinated may be key drivers of the disproportionate representation of unvaccinated patients in our study population of 
young and otherwise healthy adults who were hospitalized with COVID-19.

There are limitations to our study. Results of our study may include incidental findings of COVID-19 among hospitalized patients. However, in our cohort, less than $25 \%$ of hospitalized patients did not receive any supplemental oxygen which suggests that most hospitalizations with confirmed COVID-19 in our study population were not mild or asymptomatic COVID-19 cases that may have been incidentally found. The diagnosis of ARDS, amongst other criteria, utilizes the ratio of arterial oxygen partial pressure $(\mathrm{PaO} 2)$ to fractional inspired oxygen ( $\mathrm{FiO} 2)$. $\mathrm{As} \mathrm{PaO} 2$ data was not included in our database, our study relied on the documentation of an ARDS diagnosis in the EMR. Additionally, we were unable to account for undiagnosed conditions. As such, it is possible that some patients may have been misclassified and erroneously included in our study population of individuals without underlying conditions.

\section{Conclusion}

Results of our descriptive study showed that among young adults without underlying conditions and no history of smoking, over $93 \%$ of those hospitalized were unvaccinated and over $13 \%$ of these developed severe COVID-19. These findings underscore the need for continued efforts in improving vaccine uptake, as well as the need to modify the perceived risk of COVID-19 severity even among those who clinically may not be considered as high risk for severe COVID-19 illness.

Supplementary Information The online version contains supplementary material available at https://doi.org/10.1007/s10900-022-01062-y.

Author Contributions SS, JN, CS, PE, AS: Conceptualization. SS: Formal analysis and investigation. SS, JN, CS, PE, AS Writing-original draft preparation. SS, JN, CS, PE; AS Writing- review and editing. PE Supervision.

Funding The authors did not receive support from any organization for the submitted work.

Data Availability The data that support the findings of this study are available on request from the corresponding author upon reasonable request. The data are not publicly available due to privacy and ethical restrictions.

Code Availability Not applicable.

\section{Declarations}

Conflict of interest The authors have no conflicts of interest to declare.
Ethical Approval This study was approved by the Memorial Healthcare System Institutional Review Board (IRB \# 2021.117).

Consent to Participate Not applicable.

Consent for Publication Not applicable.

\section{References}

1. Holshue, M. L., DeBolt, C., Lindquist, S., Lofy, K. H., Wiesman, J., Bruce, H., \& Tural, A. (2020). First case of 2019 novel coronavirus in the United States. New England Journal of Medicine.

2. WHO Director-General's opening remarks at the media briefing on COVID-19-11 March 2020. (2020). World Health Organization. Retrieved from https://www.who.int/dg/speeches/detail/ who-director-general-s-opening-remarks-at-the-media-brief ing-on-covid-19---11-march-2020.

3. Johns Hopkins University \& Medicine Coronavirus Resource Center: US Map. (2021). Johns Hopkins University.

4. CDC. (2021). COVID-19 Vaccinations in the United States. Centers for Disease Control and Prevention. Retrieved from https://covid.cdc.gov/covid-data-tracker/\#vaccinations_ vacc-total-admin-rate-total.

5. Sparks, G., Kirzinger, A., \& Brodie, M. (2021). KFF COVID-19 Vaccine Monitor: Profile Of The Unvaccinated. Kaiser Family Foundation. Retrieved from https://www.kff.org/coronaviruscovid-19/poll-finding/kff-covid-19-vaccine-monitor-profile-ofthe-unvaccinated/

6. Weekly Florida COVID-19 Data. (2021). Florida Department of Health. Retrieved from https://floridahealthcovid19.gov/

7. Caserotti, M., Girardi, P., Rubaltelli, E., Tasso, A., Lotto, L., \& Gavaruzzi, T. (2021). Associations of COVID-19 risk perception with vaccine hesitancy over time for Italian residents. Social Science \& Medicine., 272, 113688.

8. Garg, S. (2020). Hospitalization rates and characteristics of patients hospitalized with laboratory-confirmed coronavirus disease 2019-COVID-NET, 14 States, March 1-30, 2020. MMWR. Morbidity and Mortality Weekly Report, 69, 458.

9. Petrilli, C. M., Jones, S. A., Yang, J., Rajagopalan, H., O’Donnell, L., Chernyak, Y., \& Horwitz, L. I. (2020). Factors associated with hospital admission and critical illness among 5279 people with coronavirus disease 2019 in New York City: Prospective cohort study. $b m j, 369$.

10. Samuels, S., Niu, J., Sareli, C., \& Eckardt, P. (2021). The epidemiology and predictors of outcomes among confirmed COVID-19 cases in a large community healthcare system in South Florida. Journal of Community Health.

11. COVID-19: People with Certain Medical Conditions. (n.d.). Centers for Disease Control and Prevention. Retrieved from https:// www.cdc.gov/coronavirus/2019-ncov/need-extra-precautions/ people-with-medical-conditions.html.

12. Owusu, D., Kim, L., O'Halloran, A., Whitaker, M., Piasecki, A. M., Reingold, A., \& Ryan, P. A. (2021). Characteristics of adults aged 18-49 years without underlying conditions hospitalized with laboratory-confirmed coronavirus disease 2019 in the United States: COVID-NET-March-August 2020. Clinical Infectious Diseases, 72(5), e162-e166.

13. Disease Burden of Flu. (n.d.). Centers for Disease Control and Prevention. Retrieved from https://www.cdc.gov/flu/about/burden/ index.html

14. Zhou, F., Yu, T., Du, R., Fan, G., Liu, Y., Liu, Z., \& Gu, X. (2020). Clinical course and risk factors for mortality of adult inpatients 
with COVID-19 in Wuhan, China: A retrospective cohort study. The Lancet, 395(10229), 1054-1062.

15. Mahase, E. (2020). Covid-19: Death rate is $0.66 \%$ and increases with age, study estimates. BMJ: British Medical Journal 369.

16. Li, W., Moore, M. J., Vasilieva, N., Sui, J., Wong, S. K., Berne, M. A., \& Greenough, T. C. (2003). Angiotensin-converting enzyme 2 is a functional receptor for the SARS coronavirus. Nature, 426(6965), 450-454.

17. Spagnolo, P. A., Manson, J. E., \& Joffe, H. (2020). Sex and gender differences in health: What the COVID-19 pandemic can teach us. American College of Physicians, 173(5), 385-386.

18. Azar, K. M., Shen, Z., Romanelli, R. J., Lockhart, S. H., Smits, K., Robinson, S., \& Pressman, A. R. (2020). Disparities in outcomes among COVID-19 patients in a large health care system in California: Study examines disparities in access and outcomes for COVID-19 patients who are members of racial and ethnic minorities and socioeconomically disadvantaged groups. Health Affairs. https://doi.org/10.1377/hlthaff.2020.00598

19. Yancy, C. W. (2020). COVID-19 and African Americans. JAMA. https://doi.org/10.1001/jama.2020.6548

20. Webb Hooper, M., Nápoles, A. M., \& Pérez-Stable, E. J. (2020). COVID-19 and racial/ethnic disparities. JAMA, 323(24), 24662467. https://doi.org/10.1001/jama.2020.8598

21. Artiga, S., Garfield, R., \& Orgera, K. (2020). Communities of Color at Higher Risk for Health and Economic Challenges due to COVID-19. The Henry J. Kaiser Family Foundation. Retrieved April 17, 2020, from https://www.kff.org/disparities-policy/ issue-brief/communities-of-color-at-higher-risk-for-health-andeconomic-challenges-due-to-covid-19/.

22. Community Social \& Economic Factors-2015-19. (n.d.). FL Health Charts. Retrieved from https://flhealthcharts.com/Chart sReports/rdPage.aspx? rdReport=ChartsProfiles.CommunityC ensusProfile\&pcid $=001$.

23. Key Things to Know About COVID-19 Vaccines. (n.d.). Centers for Disease Control and Prevention. Retrieved from https://www. cdc.gov/coronavirus/2019-ncov/vaccines/keythingstoknow.html.

24. Scobie, H. M., Johnson, A. G., Suthar, A. B., Severson, R., Alden, N. B., Balter, S., \& Cadwell, B. (2021). Monitoring incidence of covid-19 cases, hospitalizations, and deaths, by vaccination status-13 US jurisdictions, April 4-July 17, 2021. Morbidity and Mortality Weekly Report., 70(37), 1284.

25. Tenforde, M. W., Kim, S. S., Lindsell, C. J., Rose, E. B., Shapiro, N. I., Files, D. C., \& Smithline, H. A. (2020). Symptom duration and risk factors for delayed return to usual health among outpatients with COVID-19 in a multistate health care systems network-United States, March-June 2020. Morbidity and Mortality Weekly Report, 69(30), 993.

Publisher's Note Springer Nature remains neutral with regard to jurisdictional claims in published maps and institutional affiliations. 